\title{
Tecnura
}

\section{Control óptimo inverso como alternativa para la regulación de un convertidor DC-DC elevador}

\author{
Inverse optimal control as an alternative to regulate a Boost DC-DC power \\ converter
}

\author{
Carlos Jesús Vega Pérez¹, Ricardo Alzate Castaño²
}

Fecha de recepción: 6 de septiembre de 2014

Fecha de aceptación: 24 de agosto de 2015

Como citar: Vega Pérez, C. J., \& Castaño, R. A. (2015). Control óptimo inverso como alternativa para la regulación de un convertidor dc-dc elevador. Revista Tecnura, 19(46), 65-78. doi:10.14483/udistrital.jour.tecnura.2015.4.a05

\section{Resumen}

El presente artículo aplica los conceptos del control óptimo inverso para regular la tensión de salida de un circuito convertidor de potencia DC-DC elevador. El control óptimo inverso define a priori una señal de control óptima y busca a posteriori el funcional que esta última minimiza, facilitando la solución del problema de control óptimo tras evitar resolver explícitamente la ecuación de Hamilton Jacobi Bellman. Se plantean las ideas generales del método, definiendo las ecuaciones necesarias para calcular la ley de control óptima inversa. Posteriormente se realiza verificación numérica en MAT$\mathrm{LAB} \otimes$ para la acción de control, operando bajo la influencia de perturbaciones en el modelo del sistema. De los resultados de simulación obtenidos se confirma la pertinencia de la técnica para manipular el comportamiento dinámico del circuito. Trabajos futuros incluyen la validación en laboratorio para las predicciones teóricas propuestas.

Palabras clave: Control óptimo, Conversión de energía, Convertidores conmutados, Métodos de optimización, Sistemas de control no lineal.

\begin{abstract}
This article presents the application of the fundamental theory of the inverse optimal control problem for regulation of the output voltage in a Boost DC-DC power converter. The inverse optimal control problem states a priori an optimal control law and a posteriori it proposes the functional cost to be minimized, avoiding the explicit solution of the Hamilton-Jacobi-Bellman equation and, therefore, making easier the solution of the optimal control problem. Some analytical developments are shown here to obtain mathematical expressions needed to perform the inverse optimal control approach on regulation of the output voltage in a Boost power converter circuit under the influence of external disturbances. Numerical routines performed in MAT$L A B \otimes$ have shown promising results of the proposed technique by achieving annihilation of the perturbation effect in the system's model. Future work is focused on experimental verification of this approach on a real prototype of the DC-DC converter.
\end{abstract}

Keywords: Energy conversion, Nonlinear control systems, Optimal control, Optimization methods, Switching converters.

\footnotetext{
Ingeniero electrónico. Estudiante de Maestría en Ingeniería Electrónica Universidad Industrial de Santander (UIS). Bucaramanga, Colombia. Contacto: carlos.vega1@correo.uis.edu.co

2 Ingeniero electrónico, magister en Automatización Industrial, doctor en Automatización. Profesor Asistente en la Escuela de Ingenierías Eléctrica, Electrónica y de Telecomunicaciones (E3T) Universidad Industrial de Santander (UIS). Bucaramanga, Colombia. Contacto: ralzatec@uis.edu.co
} 


\section{INTRODUCCIÓN}

La desmedida demanda actual de recursos energéticos por parte de una humanidad creciente y con mayores requerimientos de consumo ha convertido el uso racional de la energía en uno de los aspectos más relevantes desde el punto de vista económico y ambiental para garantizar sostenibilidad (Carvalho, 2006; Garzon \& Abregu, 2009; Ikeyama, Watanabe, Isobe, \& Takahashi, 2011). En este contexto, cualquier esfuerzo por mejorar el aprovechamiento energético por parte de los sistemas de ingeniería es importante para contribuir con esta causa. Analizando el caso particular de la gestión energética, se requiere de una mayor y mejor transferencia de potencia desde la fuente principal hacia las cargas, garantizando que dicha energía en un mayor porcentaje se convierta en trabajo y asimismo se reduzcan al mínimo (idealmente cero) las pérdidas por disipación. Al dispositivo o sistema encargado de realizar esta labor se le denomina convertidor de potencia (Shepherd \& Zhang, 2004; Basso, 2008), y en aplicaciones de circuitos eléctricos posee la característica de regular los niveles de tensión y corriente entregados a una carga, a partir de la conmutación controlada de un dispositivo electrónico (en general un tiristor).

Cualquiera sea el caso, se requiere una forma de onda específica en las cargas, con la menor pérdida de energía respecto a la fuente de suministro principal. La técnica más utilizada en aplicaciones para manipular un circuito convertidor de potencia corresponde a la modificación conveniente empleando técnicas de control del ciclo útil de una señal modulada en ancho de pulso (Pulse Width Modulation, PWM) (Shepherd \& Zhang, 2004; Basso, 2008). En general, esta regulación se logra cambiando el patrón de conmutación de los dispositivos electrónicos asociados al circuito convertidor.

Entre las alternativas reportadas en la literatura para estudiar el control de un convertidor de potencia pueden listarse soluciones clásicas tradicionales, que van desde controladores PID (Arikatla \& Abu-Qahouq, 2010; Lindiya, Palani, \& lyyappan,
2012; Wang, 2013) y compensadores en el dominio de la frecuencia, hasta estrategias en el espacio de estados (Abbas, Abouchi, \& Pillonnet, 2010; Chiu, 2012; Alsmadi, Utkin, \& Xu, 2013). Adicionalmente, particular interés despierta en la comunidad académica el mejoramiento del desempeño del convertidor para compensar las pérdidas por conmutación. A este respecto, las técnicas de control basadas en consideraciones energéticas y de control de Lyapunov ofrecen una alternativa interesante (Zhou, Khambadkone, \& Kong, 2009; Seleme, Ferreira, Rezende, \& Borges, 2013; Dash \& Nayak, 2014). También lo son las técnicas de optimización, las cuales buscan reducir un funcional de costo que se relaciona con una función de energía (Geyer, Papafotiou, Frasca, \& Morari, 2008; Alasooly, 2010; Bianchi, Egea-Alvarez, Junyent-Ferre, \& Gomis-Bellmunt, 2012).

Sin embargo, en un problema de control óptimo tradicional existe una alta complejidad asociada a la determinación formal (i.e. que exista y que sea estable) para la ley de control óptima, la cual depende de las condiciones particulares del problema en consideración. Más aún, en términos matemáticos esta clase de problemas implica obtener soluciones analíticas para la ecuación hamiltoniana o la ecuación de Hamilton Jacobi Bellman (Sieniutycz, 2007; Xia, Chen, \& Sun, 2011; Rivera, Chavira, Loukianov, Ortega, \& Raygoza, 2014), siendo en ciertos casos inviables o simplemente inexistentes, como puede ocurrir por cuenta de no linealidades asociadas a modelos. Por tanto, para solucionar problemas de control óptimo sin necesidad de resolver tales ecuaciones, se propone como alternativa el denominado control óptimo inverso (Freeman \& Kokotovic, 1996; Sepulchre, Jankovic, \& Kokotovic, 1996).

En un problema de control óptimo inverso, se supone a priori una ley de control óptima para la cual se determina, a posteriori, el funcional de costo a minimizar. Este enfoque alternativo ha demostrado su potencialidad para solucionar problemas en aplicaciones en ciencias e ingeniería (Kanazawa, Nakaura, \& Sampei, 2009; Aghasadeghi, Long, \& 
Bretl, 2012; Leon, Alanisb, Sanchez, Ornelas, \& Ruiz, 2012; Park, 2013). Sin embargo, son pocos los resultados reportados en la literatura referidos al control óptimo inverso para regulación de circuitos convertidores de potencia. Algunos trabajos incluyen desarrollos analíticos para sistemas discretos, como los de Ornelas, Sanchez, \& Loukianov (2010); Ornelas, Rico, Sanchez, Zuñiga, \& Casarrubias (2012); Ornelas, Sánchez, Loukianov, \& Rico (2014) y sus referencias asociadas, además de aplicaciones como las registradas en Liu, Zhang, Yang, Shi, \& He (2014); Zhang, Yang, Liu, Zeng, \& Xu (2013); Pahlevaninezhad et al. (2012).

Por tanto, el presente artículo propone, como principal contribución, la formulación de un problema de control óptimo inverso de tiempo continuo para mantener regulada la tensión de salida de un convertidor de potencia DC-DC elevador (tipo Boost) sometido a la influencia de perturbaciones, como caso de aplicación para esta técnica alternativa de control óptimo en el contexto de otros métodos basados en técnicas hamiltonianas o de regulación cuadrática lineal. El contenido del artículo se presenta como sigue: la sección 2 introduce el problema de control óptimo inverso; la sección 3 ilustra la metodología empleada para aplicar control óptimo inverso en un convertidor de potencia DC-DC elevador; la sección 4 muestra los resultados obtenidos tras realizar verificación

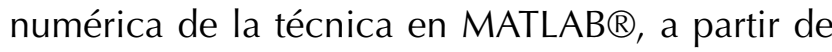
los cuales se formulan las conclusiones presentadas en la sección 5 .

\section{EL PROBLEMA DE CONTROL ÓPTIMO INVERSO}

Consideremos el sistema dinámico en tiempo continuo dado por la ecuación (1).

$\dot{x}(t)=f(x(t))+g(x(t)) u(t), \quad x_{o}=x(0)$,

donde $x(t) \in \Re^{n}$ es el vector de variables de estado en función del tiempo; $u(t) \in \mathfrak{R}^{m}$ es el vector de entradas correspondiente con las señales de control, $f: \Re^{n} \rightarrow \Re^{n} y g: \Re^{n} \rightarrow \Re^{n x m}$ son funciones, en general, no lineales del estado.

A partir de ello, un problema de control óptimo para este sistema se define como la manera de encontrar una ley de control óptima $u(t)=u^{*}$, que permita minimizar (o maximizar, dependiendo de las condiciones del problema) el índice de desempeño dado por la ecuación (2).

$$
J=\int_{0}^{\infty}\left(l(x(t))+u^{T}(t) R(x(t)) u(t)\right) d t
$$

Donde $l(x(t)) \geq 0$ y $R(x(t))>0$ para todo $x(t)$. Para hallar esta ley de control óptima $u^{*}$ es condición suficiente aplicar el siguiente teorema (Sepulchre, Jankovic, \& Kokotovic, 1996):

\section{Teorema 1: (Condición suficiente para la optimalidad)}

Si existe una función semidefinida positiva $V(x)$ en $C^{1}$, que satisface la ecuación (3) o de Hamilton Jacobi Bellman (HJB).

$$
l(x)+\frac{\partial V}{\partial x} f(x)-\frac{1}{4} \frac{\partial V}{\partial x} g(x) R^{-1}(x) g^{T}(x) \frac{\partial V}{\partial x}=0, \quad V(0)=0,
$$

donde $l(x)$ y $R^{-1}(x)$ vienen dados por el funcional de costo de la ecuación (2), mientras $f(x)$ y $g(x)$ por el sistema de la ecuación (1), de tal manera que el control de retroalimentación es definido por la ecuación (4).

$$
u^{*}=-\frac{1}{2} R^{-1}(x) g^{T}(x) \frac{\partial V}{\partial x},
$$

entonces, si $u^{*}$ logra la estabilidad asintótica en el punto de equilibrio, será el control óptimo de estabilización que minimiza el funcional de costo de la ecuación (2) para todo $u(t)$, garantizando $\lim _{t \rightarrow \infty} x(t)=0$ y $V(x)$ será la función de valor óptimo.

Así, para resolver el problema de control óptimo se hace necesario determinar la función $V(x)$ 
que resuelve la expresión HJB en la ecuación (3), para determinar la ley de control de realimentación estabilizante. En general, la solución explícita para dicha ecuación no es un problema trivial. Solo en algunos casos simples como el regulador cuadrático lineal (LQR) es posible derivar resultados analíticos precisos.

Por tanto, como una alternativa para encontrar la ley de control óptima estabilizante sin necesidad de resolver la correspondiente ecuación HJB, se propone el denominado control óptimo inverso. En este enfoque se establece a priori una ley de control estabilizante para el problema y a posteriori se obtiene el funcional de costo que dicha ley de control minimiza (Sepulchre, Jankovic, \& Kokotovic, 1996).

Inicialmente, se define a partir del problema de estabilización dado por la ecuación (4), la ley de control óptima inversa $u^{*}$, dada como en la ecuación (5).

$$
u^{*}=-\frac{1}{2} R^{-1}(z) g^{T}(x) \frac{\partial V}{\partial z}, \quad R(z)>0,
$$

Donde se incorpora como variable de interés el error $z=x-x_{d}$ (siendo $x_{d}$ los valores deseados de $x$ ), para constituir un problema de seguimiento de trayectorias. Luego, asumiendo una forma cuadrática para $V(z)$ a partir de la ecuación (6).

$$
(z)=\frac{1}{2} z^{T} P z, \quad P=P^{T}>0,
$$

La ley de control toma la forma de la ecuación (7).

$$
\begin{gathered}
u^{*}=-\frac{1}{4} R^{-1}(z) g^{T}(x) \frac{\partial\left(z^{T} P z\right)}{\partial z} \\
u^{*}=-\frac{1}{2} R^{-1}(z) g^{T}(x) P
\end{gathered}
$$

Donde $P=P^{T}>0$ y $R(z)=R(z)^{T}>0$. A partir de esta la ley de control se define el funcional de costo asociado a este problema de optimización tomando en cuenta que $V(z)$ es una función de Lyapunov y, por tanto, para estabilidad se requiere que (Sepulchre, Jankovic, \& Kokotovic, 1996).

$$
\dot{V}=\frac{\partial V}{\partial z} f(x)+\frac{\partial V}{\partial z} g(x) u \leq 0, \quad u=\frac{1}{2} u^{*}
$$

Obteniendo a partir de ello la ecuación (8).

$$
\dot{V}=\frac{\partial V}{\partial z} f(x)-\frac{1}{4} \frac{\partial V}{\partial z} g(x) R^{-1}(z) g^{T}(x) P z \leq 0
$$

Lo anterior permite definir la ecuación (9) para $l(z)$, que constituye una solución para (es decir, que anula) la ecuación HJB en (3).

$$
l(z):=-\dot{V}=-\frac{\partial V}{\partial z} f(x)+\frac{1}{4} \frac{\partial V}{\partial z} g(x) R^{-1}(z) g^{T}(x) P z
$$

En resumen, el problema de control óptimo inverso consiste en que a partir de la definición de la ley de control óptimo en la ecuación (7), la cual a su vez depende de la función $V(z)$ propuesta en la ecuación (6), el funcional de costo en la ecuación (2) se minimiza siempre que $l(z)$ corresponda con la ecuación (9).

\section{METODOLOGÍA}

A continuación se realizará la aplicación de los conceptos anteriormente enunciados respecto al control óptimo inverso en la manipulación del comportamiento dinámico de un circuito convertidor de potencia. Se considerará el caso particular de la regulación de tensión a la salida de un convertidor tipo elevador o Boost.

\section{Modelo del circuito convertidor Boost}

El diagrama del convertidor de potencia DC-DC tipo elevador (Boost), con un conmutador ideal, se muestra en la figura 1. El convertidor Boost amplifica la tensión de entrada constante $E$ por un factor escalar relacionado con el ciclo útil.

Para obtener el modelo matemático que describe la dinámica del convertidor Boost, se aplican las leyes de Kirchoff sobre el circuito resultante tras posicionar el interruptor en modo de encendido o apagado. En adelante, estos modos de conmutación serán representados mediante la variable de control $U$ 


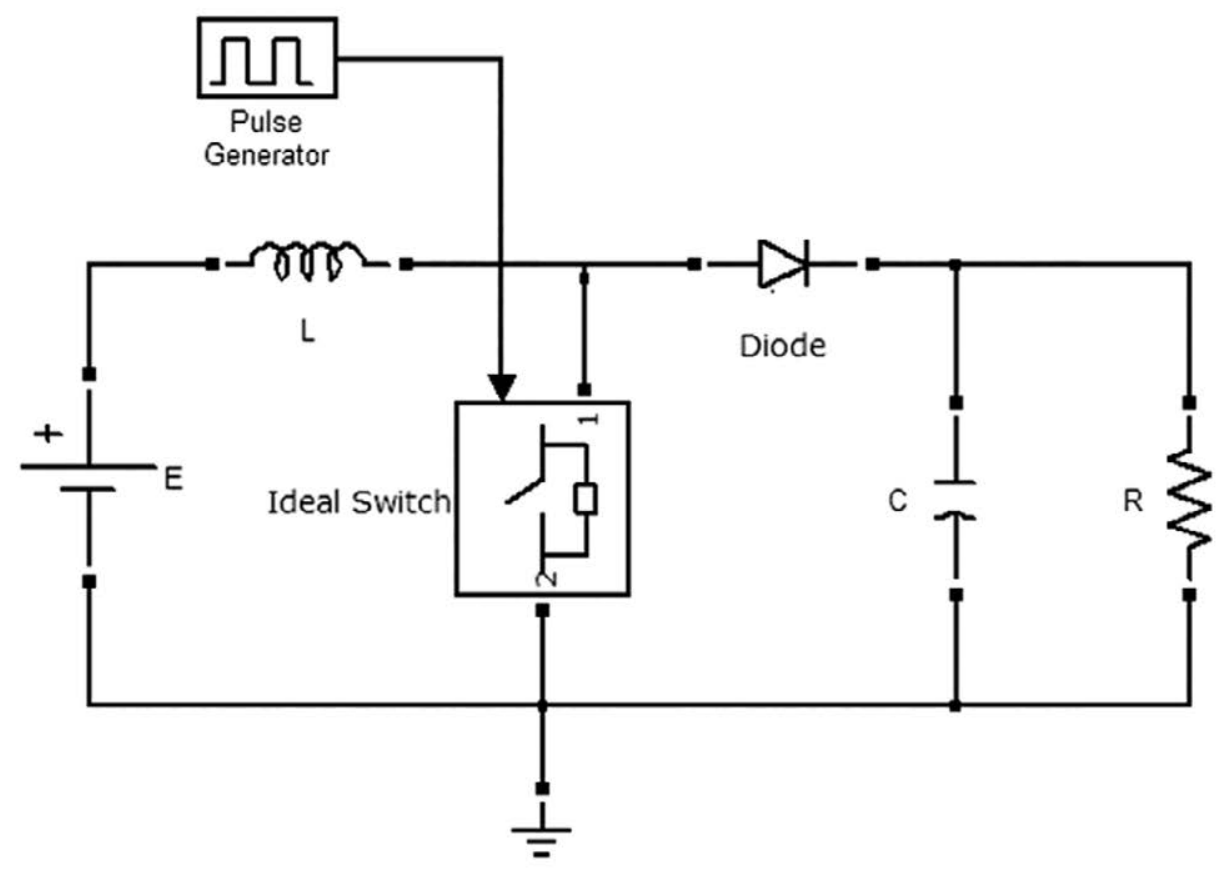

Figura 1. Representación del convertidor Boost con un conmutador ideal.

Fuente: Elaboración propia.

Inicialmente, se considera el modo de no conducción para el diodo $(U=1)$. La topología correspondiente se muestra en la figura 2 . A partir de ello, se obtiene el sistema de ecuaciones diferenciales dado por la ecuación (10).

$$
\begin{gathered}
L \frac{d i}{d t}=E, \\
C \frac{d i}{d t}=-\frac{v(t)}{R}
\end{gathered}
$$

Donde $i(t)$ representa la corriente que pasa por el inductor, al tiempo que $v(t)$ es la tensión en el capacitor. Asimismo los parámetros $R, L, \mathrm{C}$ y $E$ representan para el circuito, respectivamente, los valores de resistencia, inductancia, capacitancia y tensión de entrada constante.

Una segunda topología de circuito corresponde al modo de conducción para el diodo $(U=0)$, la cual se muestra en la figura 3. El sistema de ecuaciones diferenciales que describe este modo de operación, está dado por la ecuación (11).

$$
\begin{gathered}
L \frac{d i}{d t}=-v+E \\
C \frac{d i}{d t}=i-\frac{v}{R}
\end{gathered}
$$

Posteriormente, tras combinar las ecuaciones (10) y (11), se puede obtener el modelo del sistema unificado incorporando la acción de control $U$ de la manera presentada en la ecuación (12), donde se definen como variables de estado la corriente en el inductor $x_{1}=i(t)$ y la tensión en el condensador $x_{2}=v(t)$ siendo $U$ un valor binario que relaciona la posición del conmutador.

$$
\begin{gathered}
\dot{x}_{1}=\frac{E}{L}-\frac{x_{2}}{L} U \\
\dot{x}_{2}=-\frac{1}{R C} x_{2}+\frac{x_{1}}{C} U
\end{gathered}
$$

De otro lado, tomando en cuenta la naturaleza continua del control en la ecuación (7), se requiere 


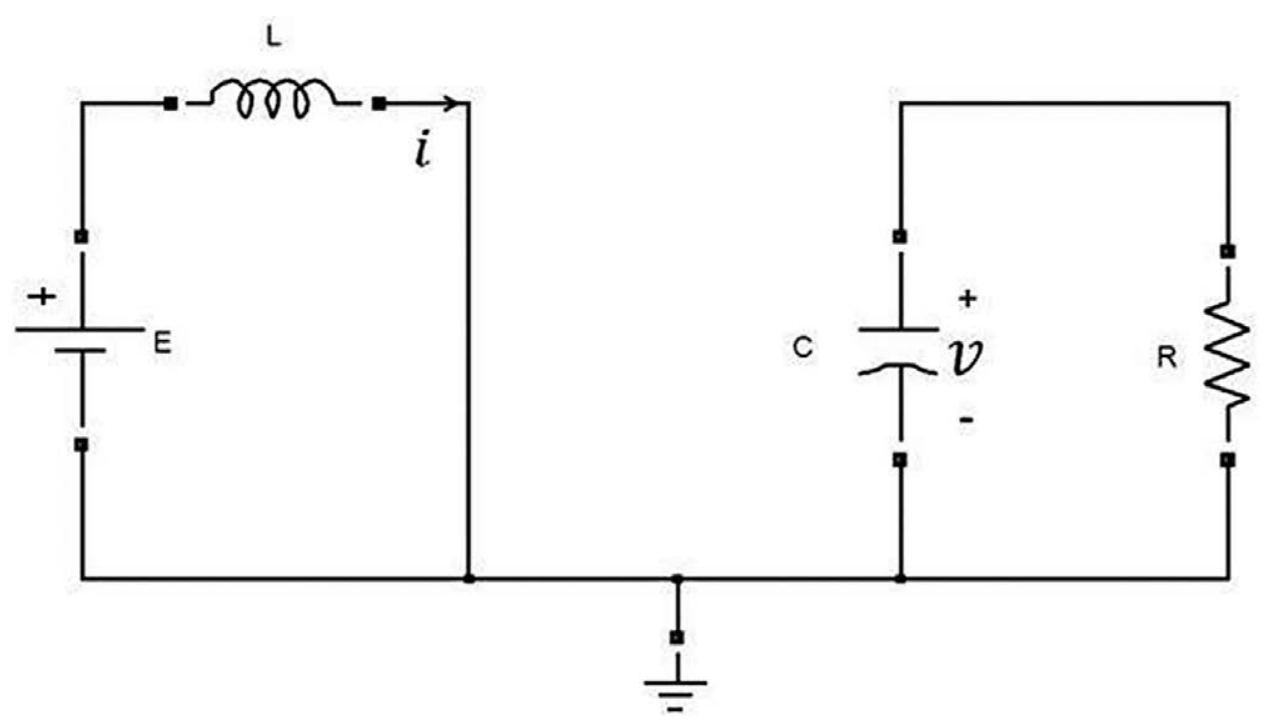

Figura 2. Representación del convertidor Boost en modo de no conducción.

Fuente: Elaboración propia.

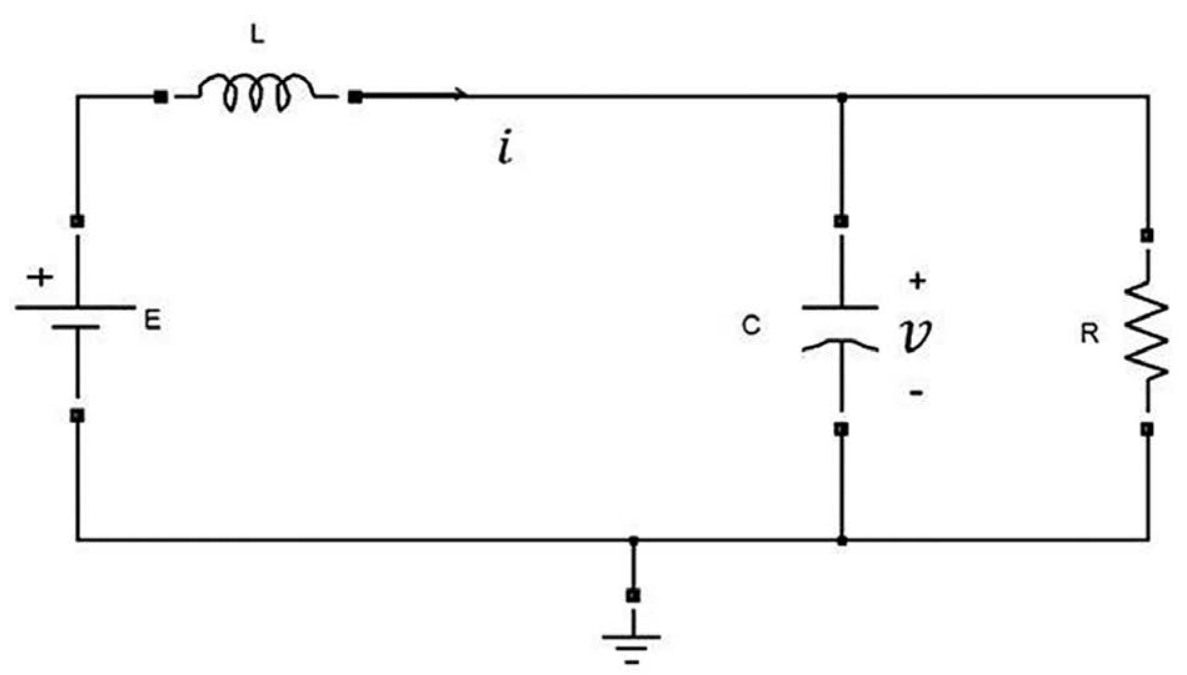

Figura 3. Representación del convertidor Boost en modo de conducción.

Fuente: Elaboración propia.

emplear un modelo promediado del sistema en la ecuación (12) válido para altas frecuencias de conmutación (Kassakian, 1991). Este modelo pro-

$$
\begin{gathered}
\dot{x}_{1}=\frac{E}{L}-\frac{x_{2}}{L} u \\
\dot{x}_{2}=-\frac{1}{R C} x_{2}+\frac{x_{1}}{C} u
\end{gathered}
$$
mediado se presenta en la ecuación (13), donde $u$ toma valores continuos en el intervalo $[0,1]$. 


\section{Regulación del voltaje de salida del circuito}

El objetivo es controlar la tensión de salida en el convertidor, por tanto inicialmente se selecciona en la ecuación (14) la tensión del condensador como salida del sistema.

$$
y=x_{2}
$$

Esta selección en la señal de salida hace al sistema de fase no mínima y en consecuencia, según la teoría, no se puede lograr en un sistema de este tipo un seguimiento de una trayectoria. Cualquier técnica de control para llevar a cabo el seguimiento exacto haría que el sistema realimentado resultante sea inestable (Kassakian, Schlecht, \& Verghese, 1991). Una forma simple de solucionar este inconveniente es controlar el voltaje indirectamente mediante el control de la corriente del inductor. Por tanto, se selecciona a esta como la nueva salida:

$$
y=x_{1}
$$

Cuando la tensión de salida es constante, la relación existente entre la corriente del inductor y la tensión en el capacitor se puede encontrar a través de los puntos de equilibrio del sistema, definidos mediante la ecuación (15).

$$
\bar{x}_{2 d}=\frac{E}{\bar{u}}, \quad \bar{x}_{1 d}=\frac{E}{\bar{u}^{2} R},
$$

Donde $\bar{x}_{1 d}, \bar{x}_{2 d}$ y $\bar{u}$ representan valores en estado estable para $x_{1}, x_{2}$ y $u$ respectivamente. Sustituyendo $\bar{u}$ obtenemos la relación dada por la ecuación (16).

$$
\bar{x}_{1 d}=\frac{\bar{x}_{2 d}^{2}}{E R}
$$

Esta última expresión permite definir un valor para la corriente deseada del circuito a partir del valor de tensión esperado en la salida del mismo, asegurando así un control en las dos direcciones del espacio de estados. De no hacerlo, podría crecer de manera incontrolada el valor de la corriente y limitar por tanto la aplicabilidad práctica de la técnica.

Para el diseño de la ley de control óptimo inverso se emplearán los parámetros del circuito dados por la tabla 1 y adicionalmente las matrices de ponderación propuestas en la ecuación (17) cuya selección corresponde con un criterio de ensayo y error.

Tabla 1. Parámetros nominales del convertidor Boost.

\begin{tabular}{ll}
\hline \multicolumn{1}{c}{ Parámetro } & \multicolumn{1}{c}{ Valor } \\
\hline Tensión de entrada E & $15[\mathrm{VDC}]$ \\
\hline Tensión de salida & $30[\mathrm{VDC}]$ \\
\hline Resistencia R & $30[\Omega]$ \\
\hline Capacitor C & $20[\mu \mathrm{F}]$ \\
\hline Inductancia L & $20[\mathrm{mH}]$ \\
\hline Frecuencia de conmutación & $40[\mathrm{kHz}]$ \\
\hline Ciclo útil PWM & $50 \%$ \\
\hline
\end{tabular}

Fuente: Elaboración propia.

$$
R(z)=[5], \quad P=\left[\begin{array}{lr}
10 & 0 \\
0 & 0,001
\end{array}\right]
$$

Por tanto, a partir de la ley de control óptima inversa dada en la ecuación (7), reescrita por comodidad en este punto:

$$
u^{*}=-\frac{1}{2} R^{-1} g^{T} P\left(x-x_{d}\right),
$$

Tomando en cuenta que, a partir de la ecuación (1) en el sistema representado por la ecuación (13) $f(x)$ y $g(x)$ toman la forma:

$$
f(x)=\left[\begin{array}{c}
\frac{E}{L} \\
-\frac{1}{R C} x_{2}
\end{array}\right]=\left[\begin{array}{c}
750 \\
-\frac{5000}{3} x_{2}
\end{array}\right] ; g(x)=\left[\begin{array}{c}
-\frac{1}{L} x_{2} \\
\frac{1}{C} x_{1}
\end{array}\right]=\left[\begin{array}{c}
-50 x_{2} \\
50000 x_{1}
\end{array}\right],
$$

La ley de control que resuelve el problema de formular mediante la ecuación (18). control óptimo inverso para el circuito se puede 


$$
u^{*}=-\frac{1}{2}[5]^{-1}\left[\begin{array}{ll}
-50 x_{2} & 50000 x_{1}
\end{array}\right]\left[\begin{array}{lr}
10 & 0 \\
0 & 0,001
\end{array}\right]\left[\begin{array}{l}
x_{1}-\frac{\bar{x}_{2 d}{ }^{2}}{E R} \\
x_{2}-\bar{x}_{2 d}
\end{array}\right]
$$

La cual cumple la condición de estabilidad formulada en (8).

Finalmente, nótese el término constante incluido en la expresión para $f(x)$. Dicho término se incluye como parte de esta función tomando en cuenta la descripción general dada en la ecuación (1), debido a que no es un factor de la acción de control $g(x)$, aunque exista a su vez una independencia en el estado del sistema.

\section{RESULTADOS}

La figura 4 presenta un diagrama de flujo del

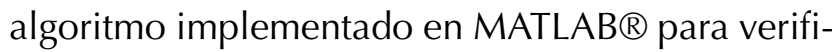
car resultados analíticos a través de simulación. En dicho diagrama, el valor mínimo de $J$ corresponde con $\left.V(z)\right|_{t=0^{\prime}}$ según reportado en la literatura (Sepulchre, Jankovic, \& Kokotovic, 1996).
El comportamiento del circuito se muestra en las figuras 5 y 6 , donde se grafican respectivamente la corriente en el inductor y el voltaje en el capacitor, a partir de simulación en MATLAB $®$ empleando los parámetros incluidos en la tabla 1. Como se observa en los resultados obtenidos en la Figura 6 , el sistema mantiene constante el valor de voltaje en su salida para ser 30 [V], desde condiciones iniciales nulas en un tiempo relativamente corto y sin oscilaciones transitorias. Este comportamiento ideal es sin embargo afectado de manera notable por una perturbación aplicada sobre la tensión de entrada del convertidor en $t=0,02[s]$. Dicha perturbación corresponde con una caída en la tensión de 3 [V]. Resultados similares se verifican para el caso de la corriente en la Figura 5. Por tanto, se

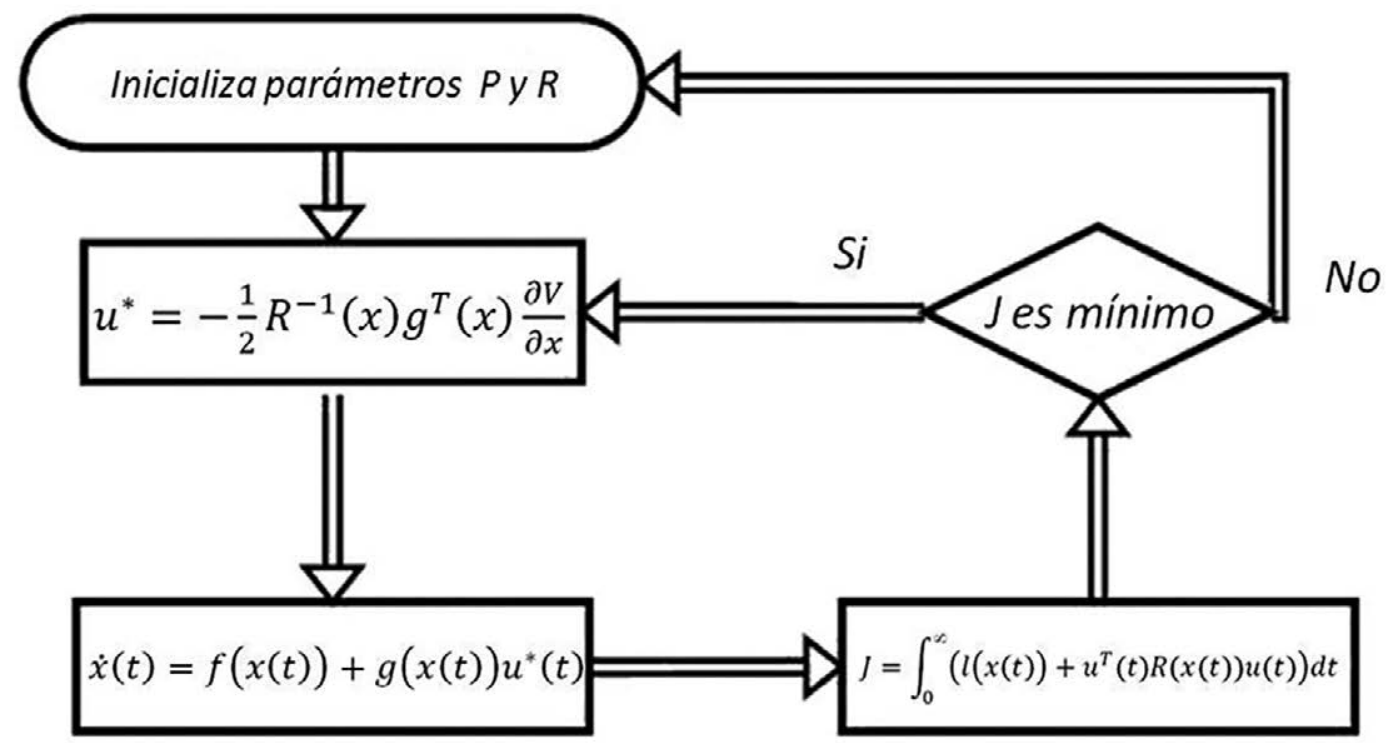

Figura 4. Diagrama de flujo del algoritmo implementado.

Fuente: Elaboración propia 


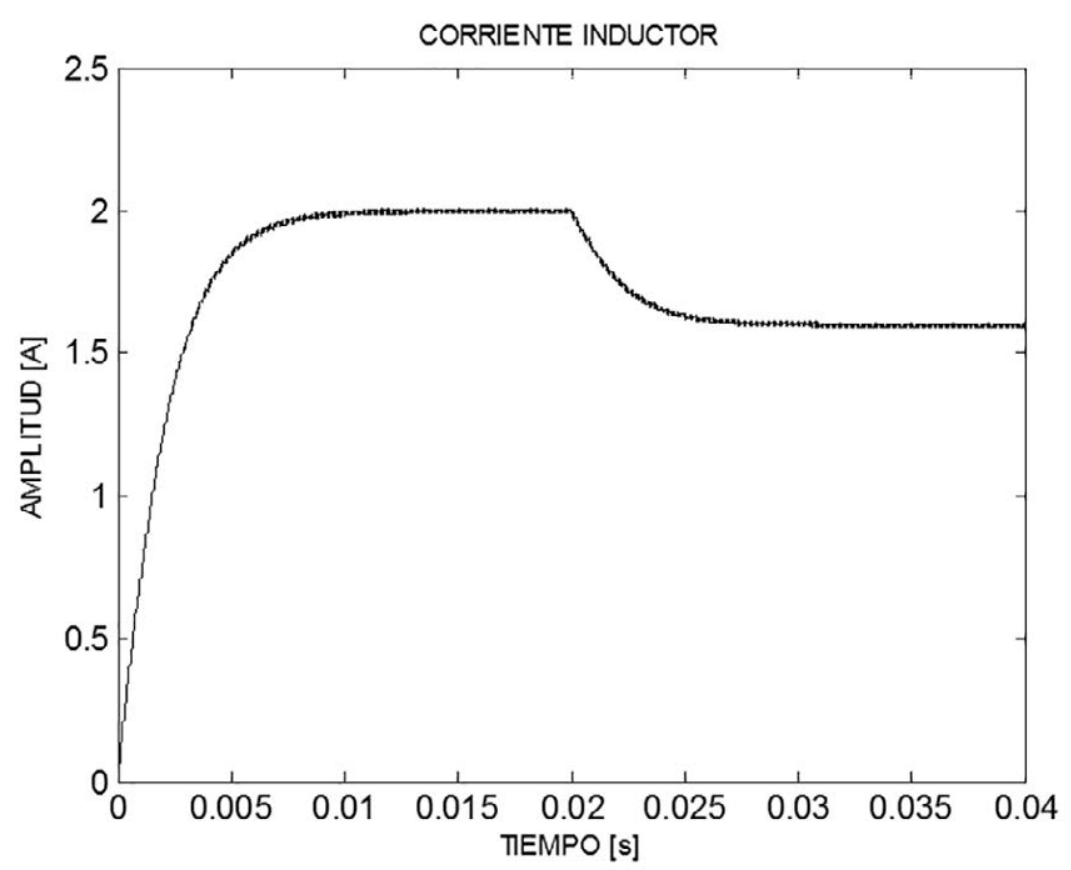

Figura 5. Corriente en el inductor del convertidor Boost en lazo abierto con perturbaciones.

Fuente: Elaboración propia

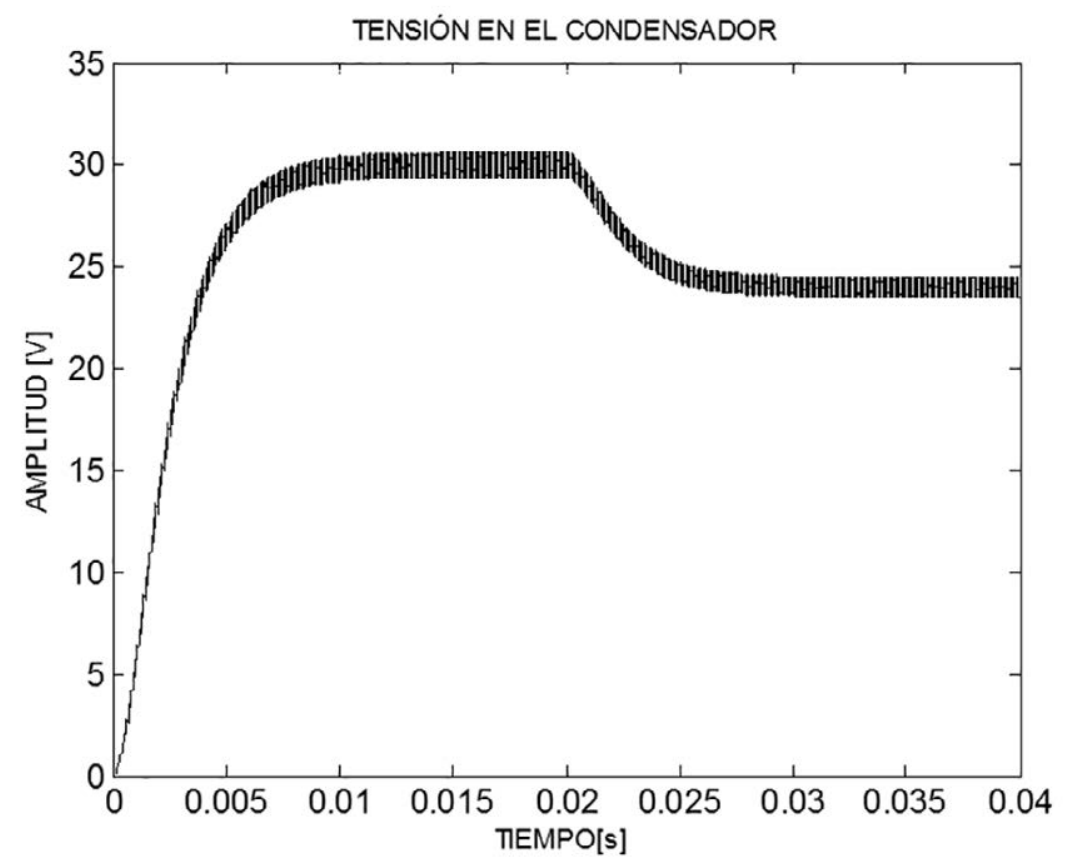

Figura 6. Tensión en el condensador del convertidor Boost en lazo abierto con perturbaciones.

Fuente: Elaboración propia 
evidencia la necesidad de aplicar un control para mantener invariante el voltaje en la salida del circuito a pesar de la acción de perturbaciones.

Una vez calculada la ley óptima de control dada por la ecuación (18), se obtienen los resultados presentados en las figuras 7 y 8 , donde se observa la trayectoria solución para los estados del sistema, que permiten verificar la regulación deseada de voltaje a la salida del circuito a pesar de la influencia de caída de tensión en la entrada. Adicionalmente, la Figura 9 permite observar la tendencia de la señal de control para converger rápidamente hacia su valor óptimo $u^{*}=50 \%$ (ciclo útil PWM), que coincide con el valor nominal reportado en la tabla 1.

De otro lado, la figura 10 presenta una verificación de la optimalidad del sistema controlado mediante (18) y a su vez un contraste para dicha medida de optimalidad con respecto a una acción de control proporcional integral (PI) convencional (no óptima), a partir de la evaluación del funcional de costo $J$ en (2). El valor teórico mínimo para este funcional es (Sepulchre, Jankovic, \& Kokotovic, 1996):

$$
\left.V(z)\right|_{t=0}=\frac{1}{2} z_{0}{ }^{T} P z_{0}=20.45,
$$

y, por tanto, se observa cómo a partir de los resultados este valor se alcanza mediante la ley de control óptimo inverso. Sin embargo, es también evidente cómo la acción de control PI convencional presenta valores no mínimos, lo cual significa desde el punto de vista físico un incremento en la energía requerida para realizar la acción de control, generando un gasto adicional innecesario que en determinadas aplicaciones (e.g. autonomía de una batería) puede marcar una diferencia importante en el desempeño del sistema.

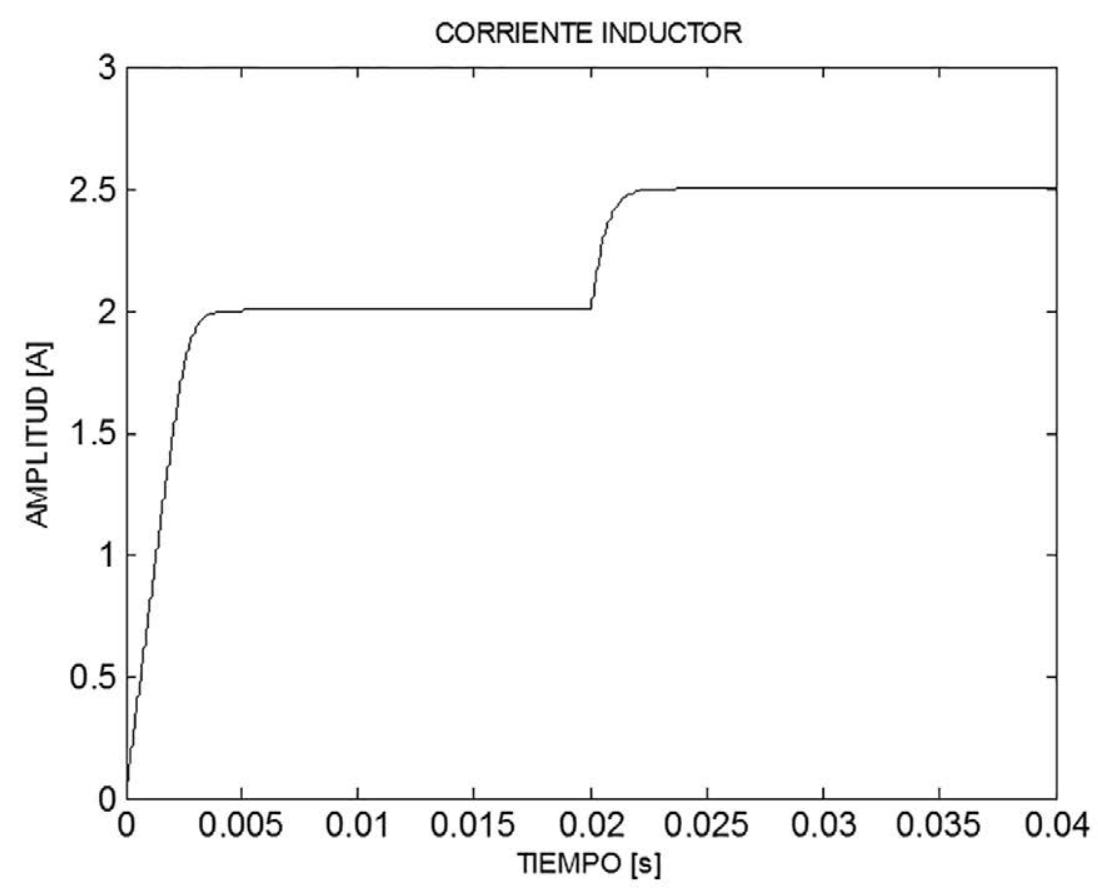

Figura 7. Corriente en el inductor del convertidor Boost.

Fuente: Elaboración propia. 


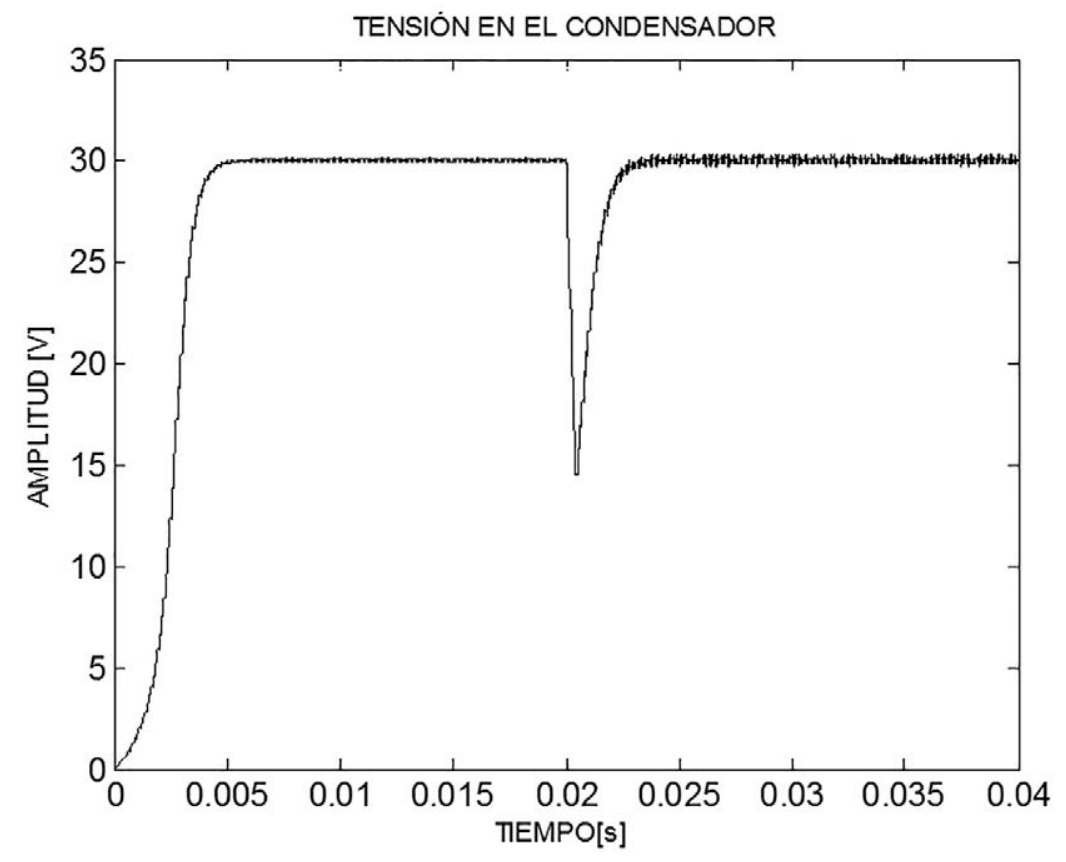

Figura 8. Tensión en el condensador del convertidor Boost.

Fuente: Elaboración propia.

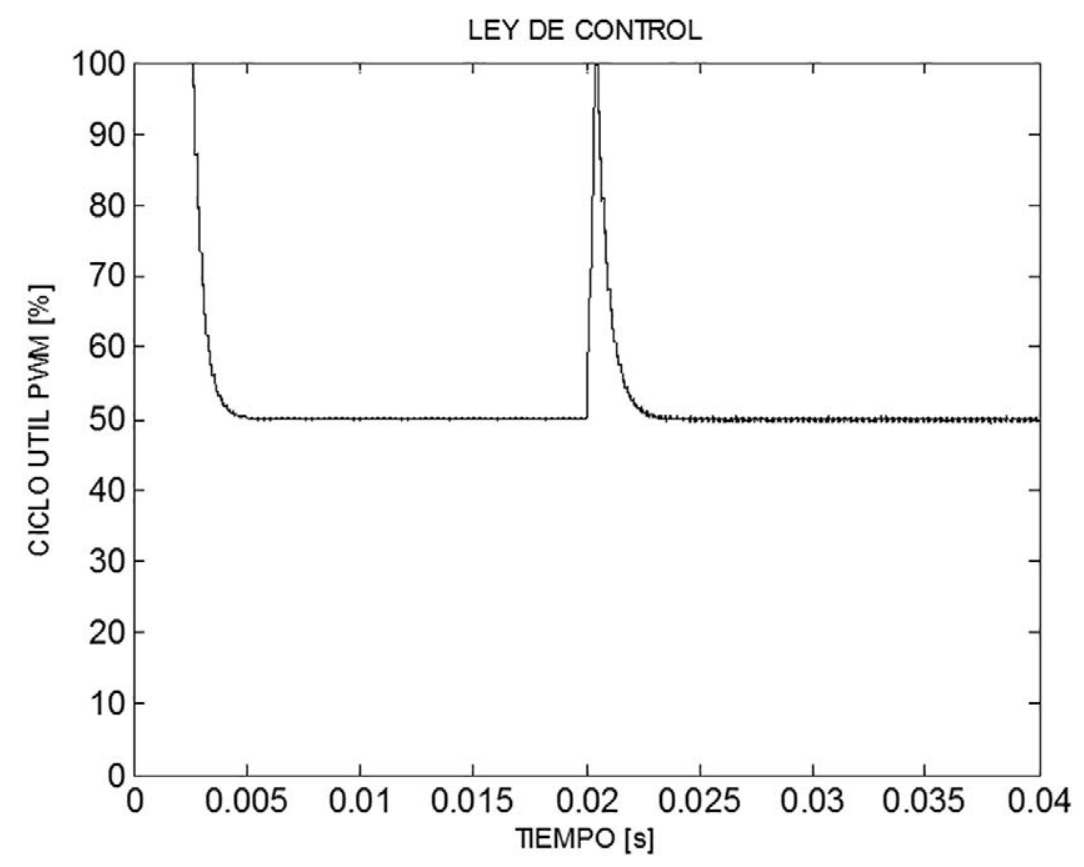

Figura 9. Ley de control del convertidor Boost.

Fuente: Elaboración propia 


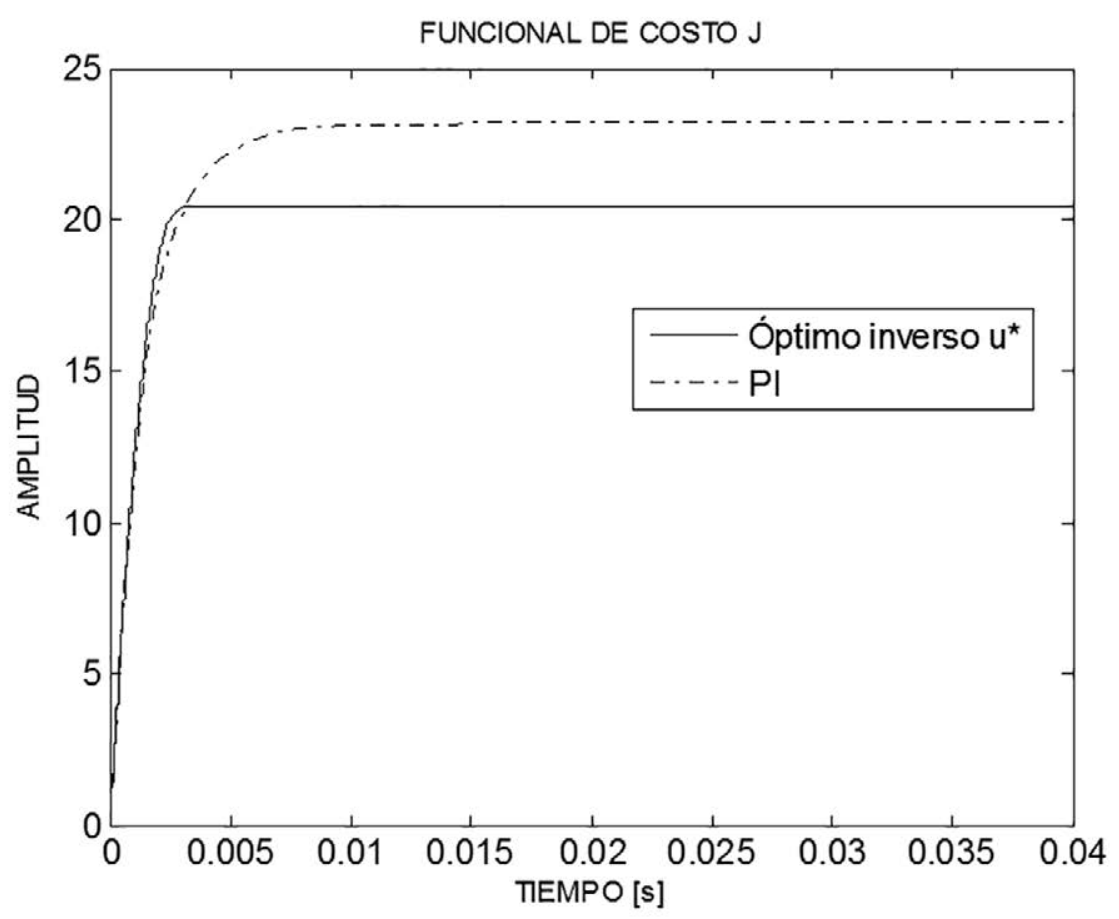

(a)

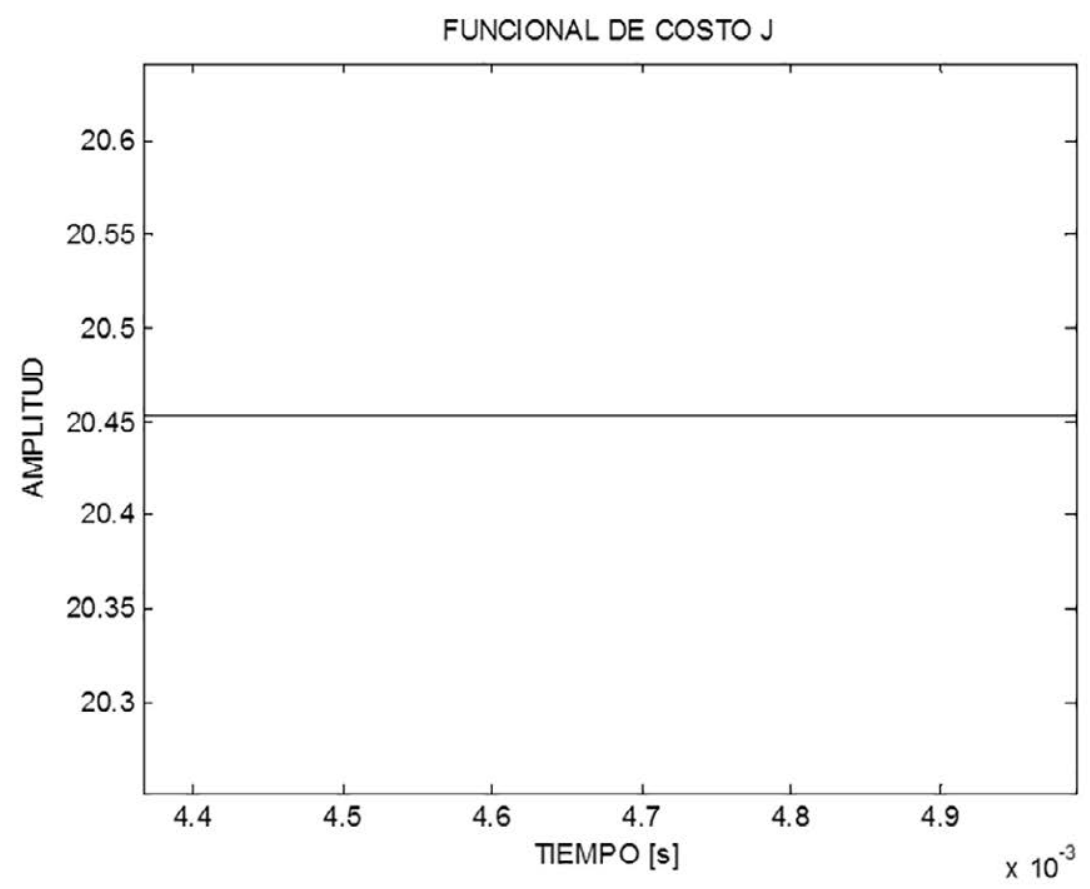

(b)

Figura 10. Funcional de costo: (a) tendencia en el tiempo sin perturbación para dos leyes de control; (b) detalle de valor para función óptima en estado estable.

Fuente: Elaboración propia. 


\section{CONCLUSIONES}

- Se han presentado, desde un punto de vista matemático y genérico, las ideas fundamentales del control óptimo inverso, del cálculo para la señal de control óptima y del funcional que esta minimiza.

- Se ha realizado un desarrollo particularizado de aplicación del control óptimo inverso, para el caso de regulación de la salida de tensión en un circuito convertidor de potencia DC-DC tipo Boost, sometido a la influencia de perturbaciones en su entrada.

- El desempeño del sistema controlado mediante la técnica propuesta ha demostrado un notorio mejoramiento en la capacidad del sistema para atenuar el efecto de las perturbaciones en su respuesta dinámica.

- La optimalidad del control se verifica a partir de corroborar el valor óptimo analítico en simulación y un posterior contraste con técnicas de control convencionales.

- Actualmente se desarrolla trabajo adicional para validar los resultados teóricos y numéricos aquí presentados, mediante prototipos experimentales manipulados en laboratorio.

\section{AGRADECIMIENTOS}

C. J. Vega desea agradecer al Programa de Jóvenes Investigadores e Innovadores de COLCIENCIAS en Colombia, por financiar el desarrollo de la presente investigación a través de la convocatoria $N^{\circ} 645$ de 2014

\section{REFERENCIAS}

Abbas, G., Abouchi, N., \& Pillonnet, G. (2010). Optimal state-space controller for power switching converter. Circuits and Systems (APCCAS), 2010 IEEE Asia Pacific Conference on, (pp. 867-870).

Aghasadeghi, N., Long, A., \& Bretl, T. (2012). Inverse optimal control for a hybrid dynamical system with impacts. Robotics and Automation (ICRA), 2012 IEEE International Conference on, (pp. 4962-4967).
Alasooly, H. (2010). Comparison between optimal control strategies applied to a system with unified power flow controller, shunt converter and series converter. Computers \& Mathematics with Applications, 60(4), pp. 954-975.

Alsmadi, Y., Utkin, V., \& Xu, L. (2013). Sliding mode control of AC/DC power converters. Power Engineering, Energy and Electrical Drives (POWERENG), 2013 Fourth International Conference on, (pp. 1229-1234).

Arikatla, V., \& Abu-Qahouq, J. (2010). An adaptive digital PID controller scheme for power converters. Energy Conversion Congress and Exposition (ECCE), 2010 IEEE, (pp. 223-227).

Basso, C. (2008). Switch-mode Power Supplies Spice Simulations and Practical Designs. New York: McGraw-Hill Professional.

Bianchi, D., Egea-Alvarez, A., Junyent-Ferre, A., \& Gomis-Bellmunt, O. (2012). Optimal control of voltage source converters under power system faults. Control Engineering Practice, 20(5), pp. 539-546.

Carvalho, J. (2006). Rational and efficient use of energy in industrial electrical installations. Universities Power Engineering Conference, 2006 UPEC '06. Proceedings of the 41st International, 3, pp. 951-955.

Chiu, C. (2012). Derivative and integral terminal sliding mode control for a class of MIMO nonlinear systems. Automatica, 48(2), pp. 316-326.

Dash, P., \& Nayak, N. (2014). Nonlinear control of voltage source converters in AC-DC power system. ISA Transactions, 53(1), pp. 1268-1285.

Freeman, R., \& Kokotovic, P. (1996). Inverse optimality in robust stabilization. SIAM Journal on Control and Optimization, 34(4), pp. 1365-1391.

Garzon, B., \& Abregu, L. (2009). Rational biomass energy use fot water heating in rural schools. Clean Electric Power, 2009 International Conference on, (pp. 128-134).

Geyer, T., Papafotiou, G., Frasca, R., \& Morari, M. (2008). Constrained optimal control of the step-down DCDC converter. IEEE Transactions on Power Electronics, 23(5), pp. 2454-2464. 
Ikeyama, T., Watanabe, H., Isobe, S., \& Takahashi, H. (2011). An approach to optimize energy use in food plants. SICE Annual Conference (SICE), 2011 Proceedings of, tpp. 1574-1579).

Kanazawa, M., Nakaura, S., \& Sampei, M. (2009). Inverse optimal control problem for bilinear systems: application to the inverted pendulum with horizontal and vertical movement. Decision and Control, 2009 held jointly with the 2009 28th Chinese Control Conference. CDC/CCC 2009. Proceedings of the 48th IEEE Conference on, (pp. 2260-2267).

Kassakian, J., Schlecht, M., \& Verghese, G. (1991). Principles of power electronics. Boston: Adison Wesley.

Leon, B., Alanisb, A., Sanchez, E., Ornelas, F., \& Ruiz, E. (2012). Inverse optimal neural control of blood glucose level for type 1 diabetes mellitus patients. Journal of the Franklin Institute, 349(5), pp. 1851-1870.

Lindiya, A., Palani, S., \& lyyappan. (2012). Performance comparison of various controllers for DC-DC synchronous Buck converter. Procedia Engineering. International Conference on Modelling, Optimization and Computing, 38, pp. 2679-2693.

Liu, X., Zhang, G., Yang, D., Shi, T., \& He, X. (2014). Discrete-time optimal control of photovoltaic grid-connected inverter based on particle swarm optimization. Hindawi Publishing Corporation. Mathematical Problems in Engineering, 2014, 10.

Ornelas, F., Rico, J., Sanchez, E., Zuñiga, G., \& Casarrubias, G. (2012). Inverse optimal trajectory tracking for discrete-time nonlinear systems: application to the boost converter. Power, Electronics and Computing (ROPEC) Conference IEEE.

Ornelas, F., Sanchez, E., \& Loukianov, A. (2010). Discrete-time inverse optimal control for nonlinear systems trajectory tracking. 49nd IEEE Conference on Decision and Control, tpp. 38-44).

Ornelas, F., Sanchez, E., Loukianov, A., \& Rico, J. (2014). Robust inverse optimal control for discrete-time nonlinear system stabilization. European Journal of Control, 20(1), pp. 38-44.

Pahlevaninezhad, M., Das, P., Servansing, A., Drobnik, J., Moschopoulos, G., Jain, P., \& Bakhshai, A.
(2012). A nonlinear optimal control approach based on the control-Lyapunov function for an AC/DC converter used in electric vehicles. IEEE Transactions on Industrial Informatics, 8(3), pp. 596-614.

Park, Y. (2013). Inverse optimal and robust nonlinear attitude control of rigid spacecraft. Aerospace Science and Technology, 28(1), pp. 257-265.

Rivera, J., Chavira, F., Loukianov, A., Ortega, S., \& Raygoza, J. (2014). Discrete-time modeling and control of a boost converter by means of a variational integrator and sliding modes. Journal of the Franklin Institute, 351(1), pp. 315-339.

Seleme, I. J., Ferreira, L., Rezende, A., \& Borges, L. (2013). Stability in passivity-based boost converter controller for power factor correction. European Journal of Control, 19(1), pp. 56-64.

Sepulchre, R., Jankovic, M., \& Kokotovic, P. (1996). Constructive nonlinear control. Berlin: Springer-Verlag.

Shepherd, W., \& Zhang, L. (2004). Power converter circuits. New York: CRC Press.

Sieniutycz, S. (2007). Hamilton-Jacobi-Bellman equations and dynamic programming for power-maximizing relaxation of radiation. International Journal of Heat and Mass Transfer, 50(13-14), pp. 2714-2732.

Wang, L. (2013). PID and predictive control of electric drives and power supplies using MATLAB/Simulink. New Jersey: Wiley.

Xia, S., Chen, L., \& Sun, F. (2011). Power-optimization of non-ideal energy converters under generalized convective heat transfer law via Hamilton-Jacobi-Bellman theory. Energy, 36(1), pp. 633-646.

Zhang, G., Yang, D., Liu, T., Zeng, Y., \& Xu, Y. (2013). Grid-connected of photovoltaic module using inverse optimal control. Advanced Materials Research, pp. 1834-1839.

Zhou, H., Khambadkone, A., \& Kong, X. (2009). Passivity-based control of an interleaved current-fed full-bridge converter with a wide operating range using the Brayton-Moser form. IEEE Transactions on Power Electronics, 24(9), pp. 2047-2056.

\section{(c) $(1) \Theta$}

